\title{
Balancing Sum Rate and TCP Throughput in OFDMA based Wireless Networks
}

\author{
Toktam Mahmoodi, Vasilis Friderikos, Oliver Holland, Hamid Aghvami \\ Centre for Telecommunications Research, King's College London, \\ Strand, London WC2R 2LS, UK \\ \{toktam.mahmoodi, vasilis.friderikos, oliver.holland, hamid.aghvami\}@kcl.ac.uk
}

\begin{abstract}
In this paper, we propose a dynamic OFDMA based subcarrier/power allocation scheme, which aims to balance the aggregate rate and the achieved TCP throughput of competing TCP flows. The proposed allocation utilizes the theoretical TCP throughput which can be accomplished in the end-to-end path. In doing so, the TCP aware scheme attempts to minimize the gap between the allocated rate and the theoretical upper bound under the system constraints. Such a technique can be of significant importance since due to its popularity, TCP is commonly used for streaming video or other multimedia applications. Numerical investigations reveal that the proposed approach, provides more balance towards the TCP throughput, and under some considerations significantly increase the fairness among competing TCP flows over end-to-end paths of different characteristics. In addition to that, it also manages to avoid starvation of TCP flows with poor channel conditions.
\end{abstract}

\section{INTRODUCTION}

Next generation wireless networks, such as Long Term Evolution (LTE) [1] and IEEE 802.16e [2], specify Orthogonal Frequency Division Multiple Access (OFDMA) as their access method. OFDMA divides the available bandwidth into multiple orthogonal subcarriers, allowing users to transmit simultaneously through allocating different subsets of the available subcarriers to different users. The resource sharing problem of subcarrier allocation, transmission power and rate distribution among users has been a prominent area of research over the past few years.

Much of the previous research works in the above mentioned area has concentrated on objectives such as maximizing the overall data rate subject to power or Bit Error Rate (BER) constraints [3]. Such a data maximization problem is more appropriate for elastic data transmission. For applications requiring a fixed data rate, the commonly used approach is to minimize the overall power consumption while satisfying the minimum rate requirements [4]. Alternative formulations do, however, consider fairness, either by prioritization using the weighted sum rate method [5], or by introducing proportional rate constraints [6]. Another possible approach is presented in [7], in which fairness is considered by maximizing the lowest achieved data rate among the user set. The research presented in [8] addresses the issue of how to provide proportional fairness in OFDMA resource allocations, based on the Nash bargaining solution. Although these research works among many others investigate the issues of fairness and Quality of Service (QoS) with respect to the allocated data rate over the wireless link, aspects as pertain to the end-to-end data transmission perspective have not been sufficiently addressed.

The Transmission Control Protocol (TCP) [9] is the prominently used transport layer protocol to achieve reliable endto-end data transfer in IP based networks. TCP, however, has shown to perform rather poorly over unreliable wireless networks [10]. More specifically, the random losses of wireless links can cause TCP to erroneously assume that these losses are due to congestion, and therefore reduce its congestion window (i.e., transmission rate) unnecessarily; this effect is particularly severe in cases where the end-to-end Round Trip Time (RTT) is high. Such factors, in addition to differences in the way different flavors of TCP react to random wireless losses, open the challenging issue of designing the TCP-aware algorithms in the wireless networks [11].

A thorough overview of a cross-layer design for resource allocation in the third generation of wireless networks is given by [12], where TCP over CDMA is also addressed. TCPaware resource allocation algorithms over a CDMA network are also studied in [13], where the objective is to maximize the throughput. The proposed algorithm in that paper uses information from the TCP state machine (i.e., slow start or congestion avoidance) to appropriately allocate the data rate at the wireless link. A joint congestion control and power allocation scheme for CDMA based wireless networks is proposed in [14], based on a generalized network utility maximization framework. In the context of IEEE 802.16, reference [15] proposes a TCP-aware allocation algorithm which estimates the bandwidth demand based on the long-term data rate, and allocates resources accordingly. Finally, variants of TCP-aware resource allocation scheme are presented in [16], and [17].

Closely related to the above works, in this paper we propose a TCP-aware resource allocation algorithm for OFDMA based access networks. To this end, we define a resource allocation framework based on the channel conditions, and investigate the difference between the allocated wireless link data rate and the optimum achievable TCP throughput, denoted as the value $D$. The definition of $D$ arises from the existence of the theoretical throughput that a TCP flow can achieve, which depends on the end-to-end RTT and the packet error probability of the corresponding flow. Thus, the allocated data rate to each endto-end flow should be determined not only by the wireless link conditions, but also by a theoretical achievable TCP throughput. To do this, we specify a TCP-aware resource 
allocation approach with the objective of minimizing the difference between allocated data rate and theoretical TCP throughput. As a result, the proposed algorithm can increase fairness among competing TCP flows. Our resource allocation problem is defined over the downlink, hence the proposed algorithm is performed at the wireless base station.

This paper is organized as follows. Section II presents the the motivation and rational of the proposed scheme together with the main contributions of this paper. In Section III we detail the system model and baseline assumptions used in the paper. In section IV, the proposed TCP-aware allocation problem is introduced, together with the associated techniques to corresponding optimization problems. Section V presents the numerical investigations of the proposed scheme. Finally, this paper concludes in Section VI.

\section{Research Motivation Via A Toy ExAmple}

Despite the fact that TCP has been initially designed for elastic applications it is currently commonly used in various popular streaming applications. It is worthwhile noting that Real Media and Windows Media, the two dominant streaming media applications, both are based on TCP streaming. In that respect, in wireless networks where resources are scarce TCP traffic for such applications should not be treated as best effort but some provision on the data rate have to be considered. In the proposed approach this provision is based on the theoretical average throughput that can be achieved by TCP, based on the specific path characteristics (i.e., RTT, packet error rate). In a sense, the proposed TCP aware scheme attempts to provide a more balanced performance towards TCP throughput that is not only an optimal resource allocation for each TCP flow, but also is a fairer distribution among TCP flows.

Figure 1 depicts a two-user scenario which can give an insight on how our proposed resource allocation scheme performs. In this figure, the vertical lines show the theoretical TCP throughput for the two users, while the discrete stem points denote the achieved data rate on wireless link by each of these two users under the channel based allocation and also under the TCP-aware scheme. As expected, a channel based resource management scheme will allocate the majority of resources to the user with better channel quality (e.g. user one in this example).

On the other hand, we propose to slightly penalize the allocated data rate to this user, thereby, the aggregate of TCP flows' data rate get close to their end-to-end capacity. To examine the level of fairness among these two TCP flows, we use Jain's fairness index [18], denoted by FI. This index is well-used as a quantitative measure of fairness in both wired and wireless networks. Given $x_{i}$ the data rate of user $i$, proportional to the optimal rate that can be achieved on the corresponding end-to-end path, then $F I$ as described in Equation (1) can be the measure of fairness among end-to-

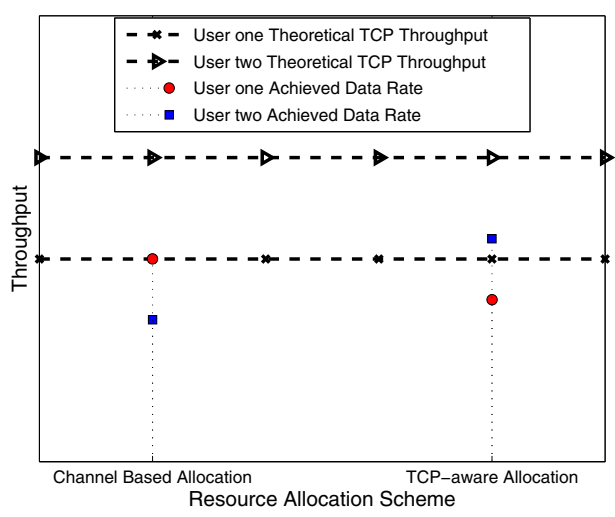

Figure 1. A comparison between channel based resource allocation and our proposed TCP-aware resource allocation

end flows.

$$
F I=\frac{\left(\sum_{i=1}^{n} x_{i}\right)^{2}}{n \cdot \sum_{i=1}^{n} x_{i}{ }^{2}} .
$$

The index $F I$ takes the value of 1 when there is a complete fair allocation. It can be seen in Figure 1 that, $F I$ as achieved by the channel allocation scheme is 0.711 , while TCP-aware scheme can increase this value to 0.966 .

In this respect, the main contributions of the paper are as follows. A novel resource allocation scheme for OFDMA networks is developed that takes into account the theoretical average of TCP throughput. The TCP steady-state throughput is used as it is the actual capacity of the end-to-end path. Unlike available solutions in the literature, we use the closed form expression of TCP throughput [19] as a means of TCP-awareness in the allocations, and in contrast with existing TCP-aware resource allocation techniques, we focus on OFDMA-based systems. To the best of our knowledge, this is the first research work which addresses this problem. In addition, we study the performance of the proposed method, not only from the TCP point of view, but also from the wireless link side. We show that the proposed approach can also provide significantly fairer allocations among end-to-end flows compared with the existing methods which attempt to allocate OFDMA subcarriers fairly [5][6][7][8] but do not consider the effect on TCP.

\section{SySTEM MOdEL}

We assume $n$ active TCP flows all of which operate in congestion avoidance phase. A single cell OFDMA network is assumed with $m$ available subcarriers. Let for flow $i$ the rate on subcarrier $j$ to be $r_{i j}$. Each user is associated with a single TCP flow, therefore, the achievable rate for user $i$ can be written as follows,

$$
R_{i}=\sum_{j=1}^{m} a_{i j} r_{i j},
$$


$a_{i j}= \begin{cases}1 & \text { if subcarrier } j \text { is assigned to user } i \\ 0 & \text { if subcarrier } j \text { is not assigned to user } i\end{cases}$

The channel gain of user $i$ at the subcarrier $j$ is denoted by $G_{i j}$. With the thermal noise power, $\sigma^{2}$, the $i$ th user's received SNR on subcarrier $j$ is given in Equation (4), in which $p_{i j}$ expresses the allocated power to flow $i$ on subcarrier $j$.

$$
\gamma_{i j}=\frac{p_{i j} G_{i j}}{\sigma^{2}}
$$

Data rate allocation for each user is based on adaptive modulation and the associated BER for each subcarrier is expressed based on the adaptive M-array quadratic modulation (M-QAM) [20].

$$
B E R_{i j} \approx c_{1} e^{-c_{2} \frac{\gamma_{i j}}{2^{r_{i j}}-1}},
$$

where $c_{1} \approx 0.2, c_{2} \approx 1.5$.

Similar to [8] we assume a fixed BER for all users across all subcarriers $\left(B E R=B E R_{i j} \forall i, j\right)$. Solving for $r_{i j}$, the achievable rate for user $i$ on the $j$ th subcarrier can be described with Equation (6), in which $c_{3}=-\ln \left(B E R / c_{1}\right) / c_{2}$, and $w$ is the equal bandwidth of each subcarrier $j$.

$$
r_{i j}=w \log _{2}\left(1+\frac{p_{i j} G_{i j}}{\sigma^{2} c_{3}}\right) \text { bits } / s .
$$

Without loss of generality, a slow-fading channel is assumed such that the channel is constant within each OFDM frame. The slowly time varying assumption is crucial since we also ascertain that perfect estimation of the subcarriers is available for each user. Moreover, mobile users and the base station are synchronized, thus there is no inter-carrier interference.

As mentioned above, allocated subcarriers and power to user $i$ defines the achievable data rate for that user. On the other hand, the theoretical end-to-end throughput bounds the steady state data rate for each specific user. We note that the end-toend TCP throughput is not only affected by the BER of the channel, but also by the RTT.

As BER is a fixed value, TCP throughput is mainly affected by the diversity of RTT. TCP throughput of flow $i$ is expressed as a closed-form function proportional to the probability of a packet in error, which can be driven directly from BER, and the end-to-end RTT [19]. Thus, the steady state throughput of a TCP flow is denoted as,

$$
B_{i}=M S S \cdot \frac{\frac{1-p_{i}}{p_{i}}+E\left[W_{i}\right]}{\overline{R T T}_{i}\left(\frac{1}{2} \cdot E\left[W_{i}\right]+2\right)},
$$

where

$$
E\left[W_{i}\right]=-\frac{1}{3}+\sqrt{\frac{8\left(1-p_{i}\right)}{3 p_{i}}+\left(\frac{1}{3}\right)^{2}} .
$$

In Equation (7), it is assumed that there are no delayed acknowledgements and therefore every transmitted packet is acknowledged. The Maximum Segment Size of TCP is denoted by MSS, and $p$ is the probability of a TCP packet in error which depends on the BER of the wireless link. Note that $\overline{R T T}$ is the average value of RTT; thus the instantaneous variation in the RTT caused by a loss of an acknowledgement does not affect the throughput. The throughput expression here is based on the well-used TCP Reno. Although the method proposed in this paper is independent of the TCP flavor, thus the throughput expression of any other TCP flavors such as NewReno [21] can be used. The proposed scheme can also be extended to the combination of various TCP flavors.

To calculate the TCP throughput at the base station, where the resource allocation algorithms performed, the value of RTT is required. Various methods are presented in the literature to estimate RTT either actively or passively at any router in the middle of the end-to-end path. The passive measurement can be done based on the three-way handshake message [22], or by associating the data segment with the acknowledgement that triggers the packet [23]. TCP timing information can also be included in the Timestamp option of the TCP segment. Experiments show that $90 \%$ of the passive measurements are within $10 \%$ of the precise RTT value [22]. These methods are not computationally complex and can be easily implicit in the link-layer of the base station.

\section{TCP-Aaware Resource Allocation Problem}

Given $D_{i}$, the difference between allocated rate to the $i$ th user and the achievable rate by TCP of flow $i$, we attempt to maximize the overall rate while minimizing the $D_{i} \mathrm{~s}$.

$$
D_{i}=\left|\alpha \cdot B_{i}-R_{i}\right| \text {. }
$$

In Equation (9), $\alpha$ represents the proportion between the throughput at the TCP layer and at the physical layer, which is a result of TCP/IP header. Therefore, our resource allocation problem can be formulated as follows,

(P1) : Maximize $\sum_{i=1}^{n} \sum_{j=1}^{m} a_{i j} w_{j} \log _{2}\left(1+\frac{p_{i j} G_{i j}}{\sigma^{2} c_{3}}\right)-\mu \sum_{i=1}^{n} D_{i}$,

subject to:

$$
\begin{array}{rc}
\sum_{i=1}^{n} \sum_{j=1}^{m} a_{i j} p_{i j} \leq p_{t}, & \\
p_{i j} \geq 0, \quad \forall i \in\{1, \ldots, n\}, j \in\{1, \ldots, m\} \\
\sum_{i=1}^{n} a_{i j} \leq 1, \quad \forall j \in\{1, \ldots, m\} \\
a_{i j} \in\{0,1\} . \quad \forall i \in\{1, \ldots, n\}, j \in\{1, \ldots, m\}
\end{array}
$$

Constraint (10) restricts the total allocated power to the maximum available power at the base station, $p_{t}$. Constraint (12) ensures that every subcarrier is assigned to only one user.

The addressed problem is a multi objective optimization problem. There are various approaches to solve such a problem. We use the well-studied approach to combine the multiple objectives into a single objective whose solution is Pareto optimal. Therefore, the optimal solution is not unique, and it depends on the value of $\mu$. Selecting the value of $\mu$ balances the two objectives. In our problem, increasing $\mu$ can move the allocation balance towards TCP throughput, while decreasing 
$\mu$ move the balance towards data rate maximization. In Section $\mathrm{V}$, we examine how the variation of $\mu$ can affect the performance of our proposed scheme.

\section{A. Optimal Subcarrier Allocation and Power Distribution}

Clearly, subcarrier and power should be assigned jointly to achieve the optimal solution. This joint allocation represents a mixed integer non-linear programming problem which pose a high computational complexity. Although problem (P1) can be solved using well-known optimization techniques, it is prohibitive for the base station to solve this problem in real time due to its complexity.

For real-time implementation and to allow larger instances of the problem to be solved we present a greedy allocation which provides suboptimal but feasible solutions. To this end, we use the method presented in the literature [6] to decouple the problem. The addressed optimization problem can be decoupled to two separate problems. Firstly how to allocate the subcarriers, and secondly how to distribute the available power into the allocated subcarriers.

\section{B. Suboptimal Subcarrier Allocation and Power Distribution}

We use an approach similar to [6] to decouple the Previously defined optimization problem. In the subcarrier allocation it is assumed that power is equally distributed in all the subcarriers, therefore the solution is suboptimal. The principle of the algorithm is for each user to allocate the subcarrier with the highest channel gain available. We detail a suboptimal algorithm, where at each iteration, the user with the lowest value of $R_{i}-\mu D_{i}$ chooses a subcarrier; finally $\Omega_{i}$ is the set of assigned subcarriers to user $i$.

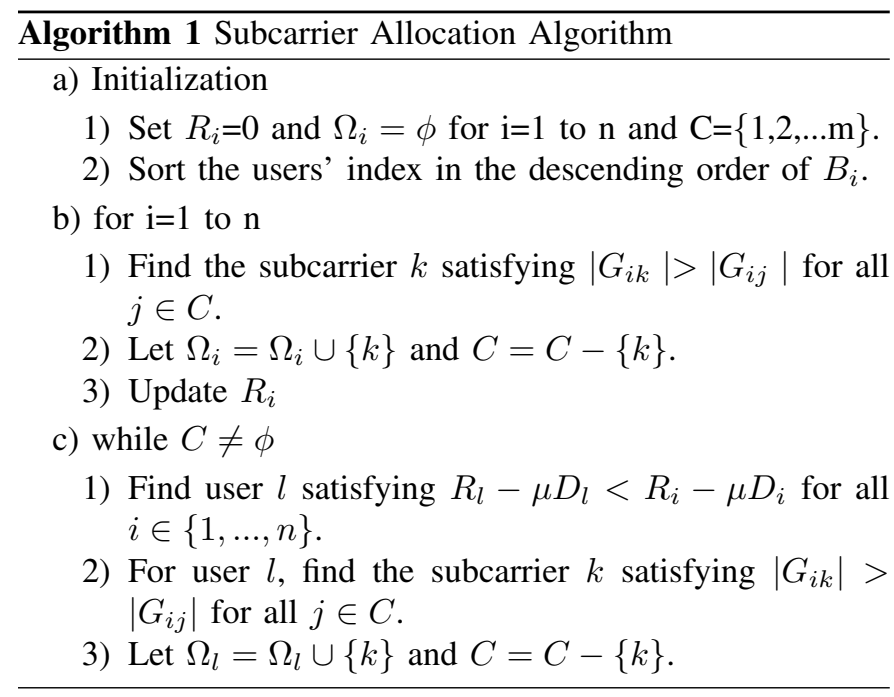

In the next step, and based on a pre-defined subcarrier allocation, problem (P1) is simplified into a maximization problem over continuous variables $p_{i j}$.

$\left(\mathrm{P}^{\prime}\right)$ : Maximize $\sum_{i=1}^{n} \sum_{j \in \Omega_{i}} w_{j} \log _{2}\left(1+\frac{p_{i j} G_{i j}}{\sigma^{2} c_{3}}\right)-\mu \sum_{i=1}^{n} D_{i}$, subject to:

$$
\begin{aligned}
\sum_{i=1}^{n} \sum_{j \in \Omega_{i}} p_{i j} & \leq p_{t} \\
p_{i j} & \geq 0 . \quad \forall i \in\{1, \ldots, n\}, j \in\{1, \ldots, m\}
\end{aligned}
$$

$\Omega_{i}$ is the set of assigned subcarriers to user $i ; \Omega_{i_{1}}$ and $\Omega_{i_{2}}$ are mutually exclusive if $i_{1} \neq i_{2}$. Therefore, Problem ( $\left.\mathrm{P}^{\prime}\right)$ can be solved using the lagrangian dual function. Differentiating the lagrangian dual function with respect to $p_{i j}$ and set the derivative to zero, power can be distributed similar to [8] and [6].

\section{Performance Investigation}

In order to investigate the performance of the proposed scheme, we consider a number of different scenarios. We simulate an OFDMA system with 52 subcarriers (equal to the number of OFDM subcarriers in IEEE 802.11a). The rest of simulation parameters are similar to the ones used in [2]. The available bandwidth is $5 \mathrm{MHz}$, maximum power at the base station is $40 \mathrm{dBm}$, and the average value of SNR is $20 \mathrm{~dB}$. The thermal noise power, $\sigma^{2}$, is $-107 \mathrm{dBm}$ (Johnson-Nyquist noise over $5 \mathrm{MHz}$ bandwidth), and the target BER is $10^{-4}$.

The MSS of the TCP flows is set to the standard maximum transfer unit of an Ethernet network which is 1460 bytes. We further assume that the end-to-end RTT for any of the TCP flows is a uniformly distributed random value in the range $[10 \mathrm{~ms}, 200 \mathrm{~ms}]$. We perform 150 Monte Carlo simulations; thus each simulation round runs with a new set of random RTT values per TCP flow, and a new set of random SNR values per subcarrier.

The iterative steps (b) and (c) in Algorithm 1 are performed to allocate subcarriers, and afterwards, in order to optimally distribute power over the allocated set of subcarriers, problem $\left(\mathrm{P}^{\prime}\right)$ is solved using the MATLAB optimization toolbox. Various values for $\mu$ are assumed, and results are compared.

Setting $\mu$ to zero converts our problem to the well-studied OFDMA resource allocation problem in the literature aiming purely to maximize sum rate; thus we use this setting as our benchmark. In our benchmark problem, the subcarrier selection is channel based, meaning that each subcarrier is allocated to the user with the highest channel gain at that subcarrier.

\section{A. Numerical Results}

Firstly, the two-user scenario described in Section II is investigated in detail in this section. Multiplier $\mu$, which balances the two objectives, is assumed to take values from zero to two (i.e. $0,0.3,0.5,0.7,1,1.5$, and 2). Since the TCP theoretical throughput of each flow is an average data rate of the corresponding end-to-end path, the utilized capacity by each TCP flow is calculated as the proportion of allocated wireless resources that can be supported by its end-to-end TCP flow.

Figure 2 presents the achieved aggregated data rate of two users on the red bar (light grey), and the differences between 


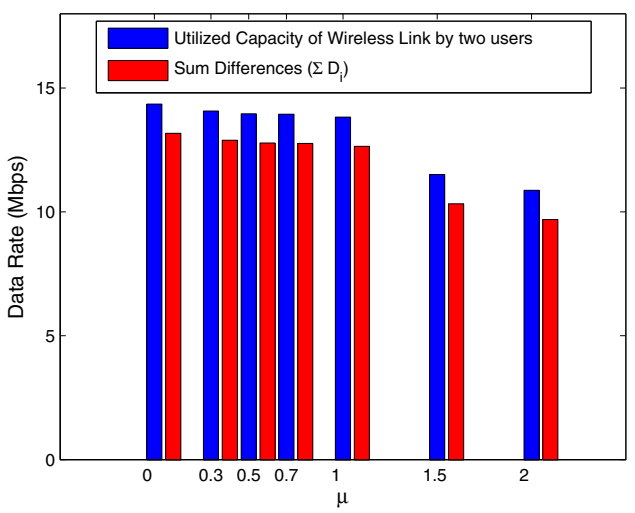

Figure 2. Two-User Scenario: Total differences between the average TCP throughput and the actual data rate on wireless link as achieved by resource allocation problem (P1) versus $\mu$, shown on the red bar (light grey bar); utilized capacity of wireless link as achieved by resource allocation problem (P1) versus $\mu$, shown on the blue bar (dark grey bar).

the allocated rate and the average TCP throughput on the blue bar (dark grey) at various values of $\mu$ over the 150 simulation rounds. It can be seen that by increasing the value of multiplier $\mu$ from 0 to 2, the aggregated achieved data rate on wireless link is decreased, while at the same time the achieved data rate gets closer to its optimal value from the TCP perspective.

In the second scenario we increase the number of mobile users to ten, with the same configuration to scenario one. Figure 3 shows the total differences between the achieved data rate by each TCP flow and its corresponding theoretical throughput (on the red/light grey bar), and the achieved aggregated data rate on the wireless link (on the blue/dark grey bar) for various values of multiplier $\mu$ in this scenario. Similar observation to scenario one can be seen in Figure 3. Across the range of values for multiplier $\mu$, the utilized capacity of wireless network is decreased by $14 \%$, although the overall achieved data rate is $20 \%$ closer to the average end-to-end capacity, which is defined by theoretical TCP throughput.

We further investigate the effect of our proposed scheme on the level of fairness among competing TCP flows. The introduced index of fairness in section II, $F I$, is calculated for these simulated scenarios, and results can be seen in Figure 4. Observing from this figure, when multiplier $\mu$ takes the value of 0.3 , the fairness index has its maximum improvements, although across various values of $\mu$, fairness among TCP flows is increased comparing with the benchmark problem $(\mu=0)$. Hence, from our studied simulation scenarios, the recommended value of balancing two objectives in the optimization problem (P1) is 0.3 , which improve the overall performance i.e. sum rate versus fairness, more significantly. Thus, we further examine the overall system performance parameters for $\mu$ equal to 0.3 , and their comparison with the benchmark problem. These results are ummarized in Tables I, and II.

In Table I, it can be seen that by increasing the value of

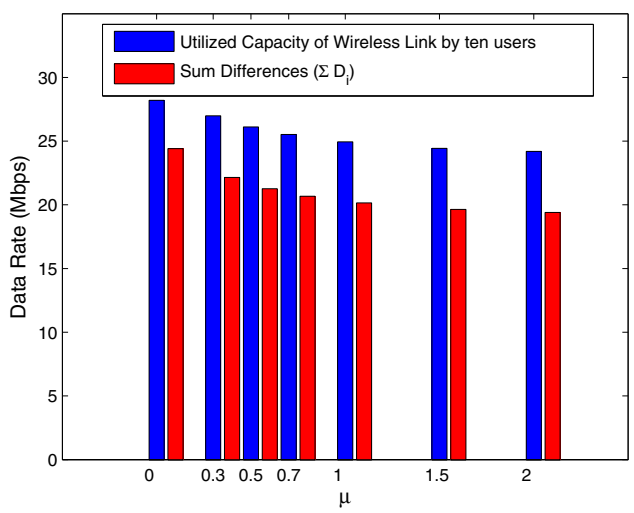

Figure 3. Ten-User Scenario: Total differences between the average TCP throughput and actual data rate on wireless link as achieved by resource allocation problem (P1) versus $\mu$, shown on the red bar (light grey bar); utilized capacity of wireless link as achieved by resource allocation problem (P1) versus $\mu$, shown on the blue bar (dark grey bar).

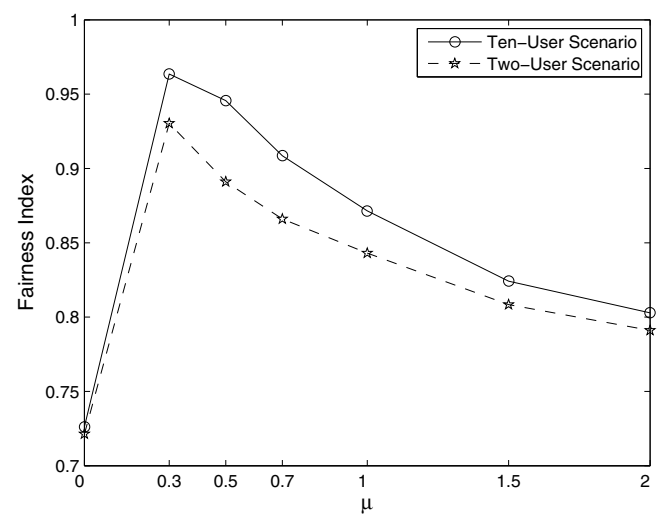

Figure 4. Ten-User Scenario: Mean Value of fairness index as achieved by resource allocation problem (P1) versus $\mu$, shown on the left axis; utilized capacity of wireless link as achieved by resource allocation problem (P1) versus $\mu$, shown on the right axis.

$\mu$ from 0 to 0.3 , the average fairness index among two users is increased by approximately $30 \%$, which can be deemed as a significant enhancement in the level of fairness among the TCP flows. At the same time, comparing the results for these two values of $\mu$, the average utilized capacity on wireless link is decreased by less than $2 \%$, and the difference of achieved data rate with the theoretical TCP throughput is decreased by $2 \%$.

Similarly, in the ten-user scenario, the improvement of $30 \%$ and $45 \%$ can be seen in the average and minimum values of the fairness index by increasing $\mu$ from 0 to 0.3 . At the same time, we can see that the utilized capacity on the wireless link is decreased by less than $5 \%$, and the achieved data rate over the wireless channel is $10 \%$ closer to the theoretical TCP throughput. 
TABLE I

Two-User Scenario: AVERAge (Mean), AND STANDARD DEVIATION (STD) VALUES OF THE SUM RATE, DIFFERENCE INDICES, AND FAIRNESS INDICES AS ACHIEVED BY THE RESOURCE ALLOCATION PROBLEM (P1) FOR $\mu$ EQUAL TO 0, AND 0.3.

\begin{tabular}{|l|c|c|}
\hline Parameter & $\mu=0$ & $\mu=0.3$ \\
\hline Mean $\left(\sum_{i=1}^{n} R_{i}\right)$ & $14.35 \mathrm{Mbps}$ & $14.07 \mathrm{Mbps}$ \\
\hline $\operatorname{std}\left(\sum_{i=1}^{n} R_{i}\right)$ & $0.624 \mathrm{Mbps}$ & $0.624 \mathrm{Mbps}$ \\
\hline $\operatorname{Mean}\left(\sum_{i=1}^{n} D_{i}\right)$ & $13.17 \mathrm{Mbps}$ & $12.89 \mathrm{Mbps}$ \\
\hline $\operatorname{std}\left(\sum_{i=1}^{n} D_{i}\right)$ & $1.102 \mathrm{Mbps}$ & $1.802 \mathrm{Mbps}$ \\
\hline $\operatorname{Mean}(F I)$ & 0.721 & 0.930 \\
\hline $\operatorname{std}(F I)$ & 0.124 & 0.094 \\
\hline
\end{tabular}

TABLE II

Ten-User Scenario: Average (Mean), And standard deviation (STD) VALUES OF THE SUM RATE, DIFFERENCE INDICES, AND FAIRNESS INDICES AS ACHIEVED BY THE RESOURCE ALLOCATION PROBLEM (P1) FOR $\mu$ EQUAL TO 0 , AND 0.3 .

\begin{tabular}{|l|c|c|}
\hline Parameter & $\mu=0$ & $\mu=0.3$ \\
\hline Mean $\left(\sum_{i=1}^{n} R_{i}\right)$ & $28.21 \mathrm{Mbps}$ & $26.99 \mathrm{Mbps}$ \\
\hline $\operatorname{std}\left(\sum_{i=1}^{n} R_{i}\right)$ & $0.580 \mathrm{Mbps}$ & $1.124 \mathrm{Mbps}$ \\
\hline $\operatorname{Mean}\left(\sum_{i=1}^{n} D_{i}\right)$ & $24.41 \mathrm{Mbps}$ & $22.15 \mathrm{Mbps}$ \\
\hline $\operatorname{std}\left(\sum_{i=1}^{n} D_{i}\right)$ & $1.470 \mathrm{Mbps}$ & $2.567 \mathrm{Mbps}$ \\
\hline $\operatorname{Mean}(F I)$ & 0.735 & 0.960 \\
\hline $\operatorname{std}(F I)$ & 0.085 & 0.035 \\
\hline
\end{tabular}

\section{CONCLUSiOnS}

In this paper a TCP-aware resource allocation algorithm is presented that takes into account the theoretical throughput that TCP can achieve. The proposed framework considers the end-to-end performance and the allocation aims to maximize the sum rate while at the same time provide balance towards TCP throughput. Thorough investigations are presented in this paper not only to study the effect of our resource allocation scheme on the end-to-end performance but also to examine the balance of the proposed multi objective optimization problem. Numerical investigations shows by choosing the optimum value for this multiplier (e.g. $\mu=0.3$ in our studied scenarios), fairness is increased by $45 \%$, which can be deemed as a significant enhancement in the level of fairness among the TCP flows. On the other hand, with the same simulation arrangements, the degradation in the sum capacity is approximately $5 \%$, and we can achieve $10 \%$ more balance towards TCP average throughput.

\section{ACKNOWLEDGMENT}

The work reported in this paper has formed part of the Delivery Efficiency Core Research Programme of the Virtual Centre of Excellence in Mobile \& Personal Communications, Mobile VCE, www.mobilevce.com. This research has been funded by EPSRC and by the Industrial Companies who are
Members of Mobile VCE. Fully detailed technical reports on this research are available to Industrial Members of Mobile VCE.

\section{REFERENCES}

[1] "IEEE Std. 802.16e-2005: Air Interface for Fixed and Mobile Broadband Wireless Access Systems, Physical and Medium Access Control Layers for Combined Fixed and Mobile Operation in Licensed Bands," IEEE Computer Society and IEEE Microwave Theory and Techniques Society, Feb. 2006.

[2] TR 25.814, V7.1.0, "Physical Layer Aspects for Evloved Universal Radio Access (UTRA)," 3rd Generation Partnership Project, Technical Specification Group Radio Access Network, Sep. 2006.

[3] I. C. Wong and B. L. Evans, "Optimal Downlink OFDMA Resource Allocation with Linear Complexity to Maximize Ergodic Rates," IEEE Trans. Wireless Commun., vol. 7, pp. 962-971, Feb. 2008.

[4] Z. Han, Z. Ji, and K. J. R. Liu, "Power Minimization for Multi-Cell OFDM Networks Using Distributed Non-cooperative Game Approach," Proc. IEEE GLOBECOM'04, Dallas, USA, Nov. 2004.

[5] L. Hoo, B. Halder, J. Tellado, and J. M. Cioffi, "Multiuser Transmit Optimization for Multicarrier Broadcast Channels: Asympotic FDMA capacity region and algorithms," IEEE Trans. Commun., vol. 52, pp. 922-930, Jun. 2004.

[6] Z. Shen, J. G. Andrews, and B. L. Evans, "Adaptive Resource Allocation in Multiuser OFDM Systems With Proportional Rate Constraints," IEEE Trans. Wireless Commun., vol. 4, pp. 2726-2737, Nov. 2005.

[7] W. Rhee and J. M. L. Cioffi, "Increase in Capacity of Multiuser OFDM System using Dynamic Subchannel Allocation," Proc. IEEE VTC Spring, Tokyo, Japan, May 2000.

[8] Z. Han, Z. Ji, and K. J. R. Liu, "Fair Multiuser Channel Allocation for OFDMA Networks Using Nash Bargaining Solutions and Coalitions," IEEE Trans. Commun., vol. 35, pp. 1366-1375, Aug. 2005.

[9] Information Science Institute, University of Southern California, CA, USA, "Transmission Control Protocol," IETF RFC 793, Sep. 1981.

[10] A. Chockalingam, E. Altman, J. Murthy, and R. Kumar, "Cross-layer design for optimizing TCP performance," Proc. IEEE ICC'05, vol. 5, pp. 3538-3542, Seoul, Korea, May 2005.

[11] T. Mahmoodi, V. Friderikos, O. Holland, and A. H. Aghvami, "Optimal Design of FEC for Fairness Maximisation among TCP flavours over Wireless Networks," IET Commun., to appear 2010.

[12] H. Jiang, W. Zhuang, and X. Shen, "Cross-Layer Design for Resource Allocation in 3G Wireless Networks and Beyond," IEEE Commun., vol. 43, pp. 120-126, Dec. 2005.

[13] M. Ghaderi, A. Sridharan, H. Zang, D. Towsley, and R. Cruz, "TCPAware Channel Allocation in CDMA Networks," IEEE Trans. Mobile Comput., vol. 8, pp. 14-28, Jan. 2009.

[14] M. Chiang, "Balancing Transport and Physical Layers in Wireless Multihop Networks: Jointly Optimal Congestion Control and Power Control," IEEE J Sel. Areas Commun., vol. 23, pp. 104-116, Jan. 2005.

[15] S. Kim and I. Yeom, "TCP-aware Uplink Scheduling for IEEE 802.16," IEEE Commun. Letters, vol. 11, pp. 146-148, Feb. 2007.

[16] T. Mahmoodi, V. Friderikos, O. Holland, and A. H. Aghvami, "TCPaware Resource Allocation Problem in OFDMA based Wireless Networks," Proc. IWCLD, Palma, Spain, Jun. 2009.

[17] T. Mahmoodi, V. Friderikos, and H. Aghvami, "Allowing Short-Lived TCP Sessions to Ramp-UP in Broadband Wireless Networks," Broadband Wireless Access workshop, GLOBECOM'09, Hawaii, USA, Dec. 2009.

[18] R. Jain, D. Chiu, and W. Hawe, "A Quantitative Measure Of Fairness And Discrimination For Resource Allocation In Shared Computer Systems," Tech. Rep. 301, Sep. 1984.

[19] J. Padhye, V. Firoiu, D. Towsley, and J. Kurose, "Modeling TCP Throughput: A Simple Model and its Empirical Validation," Proc. ACM SIGCOMM '98, pp. 303-314, Vancouver, Canada, Sept. 1998.

[20] S. T. Chung and A. J. Goldsmith, "Degrees of freedom in adaptive modulation: A unified view," IEEE Trans. Commun., vol. 49, pp. 15611571, Sep. 2001.

[21] S. Floyd, T. Henderson, and A. Gurtov, "The NewReno Modification to TCP's Fast Recovery Algorithm," IETF RFC 3782, Apr. 2004.

[22] H. Jiang and C. Dovrolis, "Passive Estimation of TCP Round-Trip Times," ACM Comp. Commun. Review, vol. 32, pp. 75-88, July 2002.

[23] B. Veal, K. Li, and D. Lowenthal, "New Methods for Passive Estimation of TCP Round Trip Times," ACM Comp. Commun. Review, vol. 3431, pp. 121-134, 2005. 